\title{
Linx
}

Revue des linguistes de l'université Paris X Nanterre

\section{Écriture du texte en français langue étrangère. Procédés d'intégration et de textualisation de ressources documentaires}

Thérèse Jeanneret, Raymond Capré et Dominique Vernaud

\section{(2) OpenEdition \\ Journals}

Édition électronique

URL : http://journals.openedition.org/linx/213

DOI : $10.4000 / \operatorname{linx} .213$

ISSN : 2118-9692

Éditeur

Presses universitaires de Paris Nanterre

\section{Édition imprimée}

Date de publication : 1 décembre 2004

Pagination : 163-186

ISSN : 0246-8743

\section{Référence électronique}

Thérèse Jeanneret, Raymond Capré et Dominique Vernaud, «Écriture du texte en français langue étrangère. Procédés d'intégration et de textualisation de ressources documentaires », Linx [En ligne], 51 | 2004, mis en ligne le 28 janvier 2011, consulté le 19 avril 2019. URL : http:// journals.openedition.org/linx/213; DOI : 10.4000/linx.213 


\title{
Hors-thème
}

\section{Écriture du texte en français langue étrangère. Procédés d'intégration et de textualisation de ressources documentaires.}

\author{
Thérèse Jeanneret (ILCF et IL, université de Neuchâtel) \\ Raymond Capré (EFM, université de Lausanne) \\ Dominique Vernaud (EFM, université de Lausanne)
}

\section{Objectif de la recherche}

La recherche présentée ici part de l'idée que l'enseignement de l'écriture du texte en français à un public alloglotte au niveau universitaire a le plus souvent une double visée : il s'agit à la fois de permettre à ce public de s'exprimer par écrit en français de manière à ce qu'il puisse faire face à l'ensemble des situations sociales requérant la maittrise de l'expression écrite (relations transactionnelles et interpersonnelles) et de lui permettre de mener ses études en français, c'est-à-dire de maitriser ce que nous appellerons, en suivant Bronckart et al. (1985), un discours théorique, adapté aux contraintes académiques.

Comme les cursus d'études de ce public sont très variés, que les exigences en matière d'écrit académique sont différentes selon les filières (par exemple, rhétoriques scientifiques différentes), il est impossible de proposer des travaux pratiques et des exercices adaptés à chaque cursus. C'est pourquoi on choisit en général dans les cursus de FLE à l'université de mettre l'accent sur le discours délibératif, c'est-à-dire d'entraîner la production de textes dont le but est de montrer quelle position doit se dégager face à tel ou tel problème. On considère que la maîtrise de ce type d'écrit s'apparente à celle du discours théorique.

Nous avons essayé de faire de ce parallélisme entre discours théorique et délibératif une heuristique de réflexion sur la didactique qu'il conviendrait de mettre 
en place pour permettre aux étudiants ${ }^{1}$ de développer leurs compétences d'écriture dans ces deux types d'écrit et cela nous a amenés à considérer comme centrales pour l'écriture les activités de référence à d'autres textes (sous la forme de citations, de mentions, de reformulation, etc.), ce que nous désignerons par le terme dimension intertextuelle de l'écriture ${ }^{2}$.

En effet, personne ne met en doute que la maitrise de l'écrit académique impose la maitrise de cette dimension intertextuelle. De nombreux travaux (voir notamment Boch \& Grossmann, 2001) ont été consacrés aux difficultés que pose ce recours à des textes déjà constitués (emprunts plus ou moins abusifs, paraphrases, organisations inadéquates des prises en charge énonciatives, problèmes de citations) et aux stratégies mises en place par les apprentis scripteurs pour citer et utiliser les idées pertinentes des textes de référence dans leur propre écrit.

Par ailleurs, on admet en général en didactique du texte écrit en langue étrangère (ainsi qu'en langue maternelle, d'ailleurs) qu'il est plus judicieux de faire produire des textes en partant de textes ${ }^{3}:$ de là la profusion d'exercices sur le résumé, la note de synthèse, etc. fleurissant dans les différents manuels proposant des activités rédactionnelles.

Il nous est ainsi apparu qu'il serait pertinent de proposer à nos étudiants, en même temps qu'un sujet de délibération, une série de documents leur permettant de se forger une opinion et ce faisant leur proposant du vocabulaire, des expressions idiomatiques, des schémas argumentatifs, etc. en relation directe avec le thème proposé. Les documents proposés (articles de journaux, émissions de radio, de télévision, extraits de romans, de bandes dessinées, etc.) joueraient ainsi le rôle d'univers textuel de référence pour le sujet proposé, de manière semblable au rôle joué par les textes théoriques de référence dans l'écriture académique.

Les sujets de délibération proposés dans les cours d'expression écrite, parce qu'ils sont dits de société, sont souvent abordés à la radio, dans les journaux, à la télévision. L'ensemble de ces discours sur tel ou tel thème fait partie de l'interdiscours ambiant, interdiscours que tout scripteur expérimenté utilise pour produire ses propres textes (cet interdiscours permet au scripteur expérimenté de se forger un point de vue, de s'y référer, de renvoyer à d'autres points de vue non soutenus, etc.). Pour un scripteur alloglotte, l'accès à cet interdiscours est plus problématique : parfois les scripteurs se réfèrent spontanément à l'interdiscours de leur(s) langue(s) d'origine

\footnotetext{
${ }^{1}$ La recherche dont il sera question dans cet article s'est fondée sur les travaux de 41 personnes : 34 étudiantes et 7 étudiants. C'est dire que les dénominations étudiant, scripteur, etc. désignent de fait plus souvent des femmes que des hommes.

${ }^{2}$ Nous rejoignons là l'idée de Simonin-Grumbach (1975) que le référent des textes théoriques est très souvent discursif puisque les textes théoriques, en plus d'élaborer les concepts qu'ils posent, utilisent les concepts élaborés ailleurs.

${ }^{3}$ On notera le caractère précurseur de [Reichler]-Béguelin, Denervaud \& Jespersen (1988) de ce point de vue.
} 
et ils manquent du vocabulaire, des structures grammaticales, des expressions préfabriquées en français. Parfois, leurs pratiques effectives de français ${ }^{4}$ sont très réduites et ils n'ont que peu d'accès à l'interdiscours ambiant, parfois aussi ils ne parviennent pas à percevoir l'existence même d'un discours sur tel ou tel sujet, par exemple si le sujet est trop étranger à leur culture d'origine.

L'idée, en proposant un ensemble de ressources documentaires sur les sujets de délibération, est de permettre aux scripteurs de découvrir des procédures d'accès à l'interdiscours et de contribuer ainsi à les aider dans une certaine mesure à élaborer leur propre univers sémiodiscursif de référence pour de tels sujets. Plus largement, ce projet se situe dans ce que Montredon (1995) appelle une pédagogie en contexte.

Le travail d'écriture d'un texte délibératif devient alors pour le scripteur d'une part une tâche de sélection d'idées dans les textes ressources, puis de citation, de reformulation, de paraphrase de ces textes et d'autre part une tâche de création: textualisation des contenus sélectionnés, construction de la cohésion-cohérence de l'ensemble du texte, organisation des arguments, choix des exemples, etc. L'ensemble des savoirs mentionnés ci-dessus sont de première importance pour la maitrise des discours théoriques et délibératifs.

L'objectif didactique est donc d'améliorer la didactique du texte délibératif écrit en l'articulant sur deux versants : un premier versant, relativement bien développé dans la littérature sur la didactique du texte écrit, concerne la structuration du texte à travers la construction de l'argumentation, la gestion de la cohésion-cohérence, etc. Le deuxième versant concerne le traitement du document comme ressource d'écriture. C'est ce versant qui constitue l'originalité de l'objectif et qui sera discuté ici.

Afin de cerner les problèmes rencontrés par les étudiants lorsqu'ils doivent intégrer dans leurs textes des «voix du dehors» et de développer des remédiations adaptées, nous avons mis sur pied, dans les départements de FLE des universités de Lausanne et Neuchâtel, une recherche-action permettant d'évaluer les réussites et les difficultés provoquées par une tâche de rédaction d'un texte délibératif accompagné d'une série de textes portant sur le thème de la délibération et d'une consigne d'utilisation de ces documents dans la réalisation de la tâche d'écriture. Nos objectifs étaient d'observer la manière dont les scripteurs réutiliseraient les documents fournis tant au niveau des lignes argumentatives choisies, des exemples sélectionnés que des citations faites (pour une étude spécifique sur la citation, voir Jeanneret, 2002), des reformulations ou des paraphrases, des réutilisations lexicales, etc.

\footnotetext{
4 Voir à ce sujet l'enquête faite à l'EFM de l'université de Lausanne et parue dans Gauthier \& Jeanneret (2000).
} 


\section{Déroulement de la recherche}

Nous avons tout d'abord choisi un thème, celui de l'autodéfense, qui nous a paru intéressant non seulement parce qu'il se prête bien à une discussion (dans quels cas l'autodéfense est-elle justifiable ?), mais aussi parce que c'est un sujet d'actualité et un domaine où les cultures d'origine diverses de nos étudiants peuvent enrichir le débat.

Ensuite nous avons constitué un corpus d'articles de presse sur le sujet. Sept textes ont été retenus : quatre articles tirés de la rubrique «Faits divers » de quotidiens de Suisse romande, un article un peu plus long du Courrier de l'Unesco, une interview du journal Construire (hebdomadaire rédactionnel émanant du groupe Migros), et quelques extraits du Code pénal suisse traitant de la légitime défense (voir ces textes en annexe 1). Pour chacun de ces textes, nous avons établi une fiche de travail, comportant d'une part une liste de termes et d'expressions spécifiques ou jugés difficiles, d'autre part un questionnaire de compréhension.

Dans nos trois classes ${ }^{5}$, nous avons consacré deux séances à un travail oral sur le thème de l'autodéfense. La première, de trois quarts d'heure, sans documents, a permis de présenter le travail et l'objectif général et d'organiser une discussion informelle sur le thème choisi. A la fin de cette première étape, les étudiants ont reçu les sept textes, qu'ils ont été priés de lire attentivement pour la séance de la semaine suivante. Au cours de cette deuxième séance, d'une durée d'une heure et demie cette fois, nous avons travaillé les textes, expliqué les termes, discuté des situations, à l'aide des questionnaires de compréhension relatifs à chaque texte distribués à ce momentlà. Il nous est cependant apparu que cette deuxième séance de travail oral collectif était trop ambitieuse : il a été impossible de traiter en détail les sept documents. Cela explique peut-être que certains documents aient été moins exploités que d'autres (la situation en Haïti, présentée dans le document 5, par exemple), ou que des textes aient présenté des difficultés de compréhension (les articles du Code pénal en particulier).

Enfin, nous avons proposé aux étudiants le travail de rédaction d'un texte délibératif à domicile, en leur distribuant une feuille de consignes rappelant la définition du thème, le problème à poser et à résoudre, l'utilisation à faire des documents étudiés, ainsi que la structure globale du texte à produire (annexe 2).

Nous avons reçu 41 travaux de nos étudiants : 9 du premier groupe lausannois (La), 18 du deuxième (Lb) et 14 du groupe neuchâtelois $(\mathrm{N})$. En plus de cette disparité numérique, il faut relever la relative hétérogénéité de nos publics : les trois cours ne sont pas identiques et poursuivent un objectif différent. Du point de vue de

\footnotetext{
${ }^{5}$ Deux groupes de l'École de français moderne de l'université de Lausanne, l'un d'un cours à option «Revue de presse » (La), l'autre d'un cours de langue écrite $(\mathrm{Lb})$, en première année de la filière Diplôme, le troisième d'un cours d'expression écrite (Certificat) de l'Institut de langue et de civilisation françaises de l'université de Neuchâtel (N).
} 
leurs connaissances linguistiques, si l'on se réfère à l'échelle globale du Portfolio européen des langues, nos étudiants s'échelonnent du niveau A2 («utilisateur élémentaire ») au niveau C1 ("utilisateur expérimenté »). Le groupe neuchâtelois en particulier est d'un niveau plus hétérogène et a globalement moins de compétences linguistiques que les deux groupes de Lausanne. Le contenu des trois cours est également différent: les textes de presse sont l'objet du cours du premier groupe lausannois (découverte des pratiques discursives, d'éléments grammaticaux du français journalistique, revue de presse, discussion à partir des textes, etc). Les deux autres cours portent sur l'expression écrite avec leur spécificité : l'activité de citation n'avait été entrainnée que dans le groupe $\mathrm{Lb}$, le groupe $\mathrm{N}$ avait été habitué à produire des textes à partir de modèles proposés.

\section{Survol général des travaux}

Les données quantitatives permettent de montrer dans quelle mesure les documents fournis ont été utilisés, de quelle manière ils l'ont été - par citation précise ou par allusion - et quels ont été les éléments lexicaux réutilisés dans les travaux. On trouvera ci-dessous le détail de ces informations recueillies dans les 41 copies analysées, et à propos des 7 documents fournis.

\section{Utilisation des documents}

Le tableau ci-dessous nous indique si tel document a été utilisé, sans tenir compte de plusieurs éventuelles occurrences.

A lire ainsi : le document 1 a été utilisé dans 32 travaux sur 41.

\begin{tabular}{|c|c|c|c|c|}
\hline & Lausanne a. & Lausanne b. & Neuchâtel & Total \\
\hline Nombre de travaux fournis & 9 & 18 & 14 & 41 \\
\hline Doc. 1 Se défendre, oui mais & 8 & 16 & 8 & 32 \\
\hline Doc. 2 Sophie amendée & 6 & 14 & 7 & 27 \\
\hline Doc. 3 Il tue son voisin & 3 & 10 & 4 & 17 \\
\hline Doc. 4 Il tue son voisin esp. & 4 & 15 & 5 & 24 \\
\hline Doc. 5 Haïti & 6 & 6 & 8 & 20 \\
\hline Doc. 6 Armes dangereuses & 4 & 12 & 5 & 21 \\
\hline Doc. 7 Code pénal & 9 & 13 & 4 & 26 \\
\hline
\end{tabular}

Tableau 1 : Nombre d'utilisations de chaque document

On notera que tous les documents ont été utilisés et chacun d'eux par plus de $40 \%$ des rédacteurs. On notera également que le document 1 a été utilisé par plus de $75 \%$ des scripteurs ${ }^{6}$. Ces derniers ont donc largement tiré parti des documents qui leur étaient proposés.

\footnotetext{
${ }^{6}$ L'explication de ce succès serait pertinente pour guider le choix des articles à retenir dans ce type d'activité d'écriture à partir de ressources documentaires. Nous ferons rapidement ici l'hypothèse
} 
Thérèse Jeanneret, Raymond Capré et Dominique Vernaud

Le tableau 2 nous donne le nombre total des utilisations pour chaque document. En le comparant au tableau 1, il apparait clairement que les documents ont été souvent utilisés plusieurs fois dans le même travail. Cela est vrai pour tous les documents, mais en particulier pour les documents 1, 2 et 7 .

\begin{tabular}{l|c|c|c||c|}
\hline Nombre de travaux fournis & Lausanne a. & Lausanne b. & Neuchâtel & Total \\
\hline \hline Doc. 1 Se défendre, oui mais & 9 & 18 & 14 & 41 \\
\hline Doc. 2 Sophie amendée & 15 & 27 & 11 & 53 \\
\hline Doc. 3 Il tue son voisin & 11 & 18 & 9 & 38 \\
\hline Doc. 4 Il tue son voisin esp. & 3 & 10 & 3 & 16 \\
\hline Doc. 5 Haïti & 5 & 16 & 6 & 27 \\
\hline Doc. 6 Armes dangereuses & 6 & 6 & 11 & 23 \\
\hline Doc. 7 Code pénal & 4 & 13 & 7 & 24 \\
\hline
\end{tabular}

Tableau 2 : Nombre total d'occurrences des documents

Nous avons schématiquement réduit le tableau suivant à deux types d'utilisation :

Citation correcte ou légèrement erronée introduite par des guillemets.

Allusion au document (simple allusion ou résumé ou comparaison entre documents, etc.)

\begin{tabular}{|c|c|c|c|c|}
\hline & Lausanne a. & Lausanne b. & Neuchâtel & Total \\
\hline Nombre de travaux fournis & 9 & 18 & 14 & 41 \\
\hline $\begin{array}{l}\text { Doc. } 1 \text { Se défendre, oui mais } \\
\text { Citation } \\
\text { Allusion }\end{array}$ & $\begin{array}{c}2 \\
13\end{array}$ & $\begin{array}{l}15 \\
12\end{array}$ & $\begin{array}{l}6 \\
5\end{array}$ & $\begin{array}{l}23 \\
30\end{array}$ \\
\hline $\begin{array}{l}\text { Doc. } 2 \text { Sophie amendée } \\
\text { Citation } \\
\text { Allusion }\end{array}$ & $\begin{array}{c}0 \\
11\end{array}$ & $\begin{array}{l}9 \\
9\end{array}$ & $\begin{array}{l}2 \\
7\end{array}$ & $\begin{array}{l}11 \\
27\end{array}$ \\
\hline $\begin{array}{l}\text { Doc. } 3 \text { Il tue son voisin } \\
\text { Citation } \\
\text { Allusion }\end{array}$ & $\begin{array}{l}0 \\
3\end{array}$ & $\begin{array}{l}1 \\
9\end{array}$ & $\begin{array}{l}0 \\
3\end{array}$ & $\begin{array}{c}1 \\
15\end{array}$ \\
\hline $\begin{array}{l}\text { Doc. } 4 \text { Il tue son voisin esp. } \\
\text { Citation } \\
\text { Allusion }\end{array}$ & $\begin{array}{l}0 \\
5\end{array}$ & $\begin{array}{c}6 \\
10\end{array}$ & $\begin{array}{l}2 \\
4\end{array}$ & $\begin{array}{c}8 \\
19\end{array}$ \\
\hline $\begin{array}{l}\text { Doc. } 5 \text { Haïti } \\
\text { Citation } \\
\text { Allusion }\end{array}$ & $\begin{array}{l}0 \\
6\end{array}$ & $\begin{array}{l}5 \\
1\end{array}$ & $\begin{array}{l}5 \\
6\end{array}$ & $\begin{array}{l}10 \\
13\end{array}$ \\
\hline Doc. 6 Armes dangereuses & & & & \\
\hline
\end{tabular}

que le succès de ce document est lié au fait qu'il présente à la fois des éléments narratifs de fait divers et des éléments informatifs sur la législation en Suisse à propos de l'autodéfense. 


\begin{tabular}{|c|c|c|c|c|}
\hline $\begin{array}{l}\text { Citation } \\
\text { Allusion }\end{array}$ & $\begin{array}{l}1 \\
3\end{array}$ & $\begin{array}{c}11 \\
2\end{array}$ & $\begin{array}{l}3 \\
4\end{array}$ & $\begin{array}{c}15 \\
9\end{array}$ \\
\hline $\begin{array}{l}\text { Doc. } 7 \text { Code pénal } \\
\text { Citation } \\
\text { Allusion }\end{array}$ & $\begin{array}{l}9 \\
9\end{array}$ & $\begin{array}{c}14 \\
7\end{array}$ & $\begin{array}{l}6 \\
0\end{array}$ & $\begin{array}{l}29 \\
16\end{array}$ \\
\hline
\end{tabular}

Tableau 3 : Type d'utilisation des documents

Il est frappant de remarquer que les documents 6 et 7 présentent un nombre de citations plus élevé que le nombre d'allusions, tandis que c'est l'inverse pour les cinq autres documents. Le caractère normatif du document 7 suffit probablement à expliquer qu'il soit cité : pour renvoyer au code pénal, il vaut mieux être le plus précis et le plus explicite possible. Pour le document 6 , on peut se demander si le fait qu'il s'agisse d'une interview, présentant de fait déjà une segmentation des différents propos tenus facilite le prélèvement en citation.

\section{Vocabulaire}

Dans chaque document, nous avons retenu un certain nombre de lexèmes, soit spécialisés soit peu courants, ou certaines constructions ou expressions préfabriquées : nous pensions que ces lexèmes ou constructions étaient inconnus des étudiants et nous souhaitions leur fournir de cette manière des données lexicales et syntaxiques nouvelles. La plupart de ces mots ou expressions ont fait l'objet d'une brève explication en classe. Nous accordons de l'importance à la réutilisation de ces lexèmes dans les travaux fournis parce qu'elle peut être considérée comme une trace de prise et partant d'acquisition (voir ci-dessous en V.).

On trouvera ci-dessous :

La liste des mots tirés des documents et retrouvés dans les travaux

Le nombre d'occurrences dans les travaux fournis.

\section{Vocabulaire}

Doc. 1

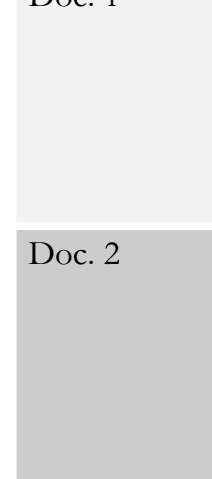

agression / agresseur

arme blanche

coup de matraque

batte de baseball

poing américain

baïonnette

à coups de poing

voies de fait

lésions corporelles

agresser

battre la chamade

gagner du temps être prévenu de lésions corporelles prohiber

se faire assaillir

tabasser

porter plainte contre recevoir une amende

infliger une amende

éloigner l'agresseur 
Thérèse Jeanneret, Raymond Capré et Dominique Vernaud

\begin{tabular}{|c|c|c|}
\hline Doc. 3 & $\begin{array}{l}\text { carabine } \\
\text { septuagénaire } \\
\text { voisin de palier } \\
\text { l'empoignade }\end{array}$ & $\begin{array}{l}\text { en détention préventive } \\
\text { importuner } \\
\text { se sentir dérangé } \\
\text { tirer sur quelqu'un }\end{array}$ \\
\hline Doc. 4 & $\begin{array}{l}\text { discriminer } \\
\text { prendre quelqu'un pour }\end{array}$ & $\begin{array}{l}\text { un individu raciste } \\
\text { un meurtrier } \\
\text { incommodé }\end{array}$ \\
\hline Doc. 5 & $\begin{array}{l}\text { les brigades de vigilance } \\
\text { les patrouilles civiles } \\
\text { les services alternatifs } \\
\text { le bidonville } \\
\text { la communauté } \\
\text { délaisser } \\
\text { lyncher, le lynchage }\end{array}$ & $\begin{array}{l}\text { se débrouiller } \\
\text { se défendre soi-même } \\
\text { être délaissé } \\
\text { délinquant, délinquance } \\
\text { corrompu } \\
\text { à défaut de }\end{array}$ \\
\hline Doc. 6 & $\begin{array}{l}\text { un abus } \\
\text { un délit } \\
\text { un profil criminel } \\
\text { commettre un délit } \\
\text { la législation }\end{array}$ & $\begin{array}{l}\text { sous strict contrôle } \\
\text { pléthore } \\
\text { une législation plus sévère } \\
\text { surarmé } \\
\text { largement répandus }\end{array}$ \\
\hline Doc. 7 & $\begin{array}{l}\text { être responsable } \\
\text { dépasser les bornes } \\
\text { repousser l'attaque l'intégrité } \\
\text { corporelle } \\
\text { pénalement }\end{array}$ & $\begin{array}{l}\text { la légitime défense } \\
\text { l'honneur } \\
\text { le patrimoine } \\
\text { les moyens proportionnés aux } \\
\text { circonstances }\end{array}$ \\
\hline
\end{tabular}

Tableau 4 : Liste des mots retrouvés dans l'ensemble des travaux

Parmi l'ensemble de ces mots, nous avons distingué les emplois corrects des emplois erronés.

\begin{tabular}{|c|c|c|c|c|}
\hline & Lausanne a. & Lausanne b. & Neuchâtel & Total \\
\hline Nombre de travaux fournis & 9 & 18 & 14 & 41 \\
\hline $\begin{array}{l}\text { Doc. } 1 \text { Se défendre, oui mais } \\
\text { correct } \\
\text { erroné }\end{array}$ & $\begin{array}{c}28 \\
2\end{array}$ & $\begin{array}{c}52 \\
2\end{array}$ & $\begin{array}{c}11 \\
0\end{array}$ & $\begin{array}{c}91 \\
4\end{array}$ \\
\hline $\begin{array}{l}\text { Doc. } 2 \text { Sophie amendée } \\
\text { correct } \\
\text { erroné }\end{array}$ & $\begin{array}{c}13 \\
3\end{array}$ & $\begin{array}{c}17 \\
2\end{array}$ & $\begin{array}{l}2 \\
1\end{array}$ & $\begin{array}{c}32 \\
6\end{array}$ \\
\hline $\begin{array}{l}\text { Doc. } 3 \text { Il tue son voisin } \\
\text { correct } \\
\text { erroné }\end{array}$ & $\begin{array}{l}7 \\
1\end{array}$ & $\begin{array}{l}9 \\
0\end{array}$ & $\begin{array}{l}2 \\
0\end{array}$ & $\begin{array}{c}18 \\
1\end{array}$ \\
\hline
\end{tabular}




\begin{tabular}{|c|c|c|c|c|}
\hline $\begin{array}{l}\text { Doc. } 4 \text { Il tue son voisin esp. } \\
\text { correct } \\
\text { erroné }\end{array}$ & $\begin{array}{l}5 \\
0\end{array}$ & $\begin{array}{c}12 \\
0\end{array}$ & $\begin{array}{l}2 \\
0\end{array}$ & $\begin{array}{c}19 \\
0\end{array}$ \\
\hline $\begin{array}{l}\text { Doc. } 5 \text { Haïti } \\
\text { correct } \\
\text { erroné }\end{array}$ & $\begin{array}{c}19 \\
2\end{array}$ & $\begin{array}{c}10 \\
0\end{array}$ & $\begin{array}{l}8 \\
0\end{array}$ & $\begin{array}{c}37 \\
2\end{array}$ \\
\hline $\begin{array}{l}\text { Doc. } 6 \text { Armes dangereuses } \\
\text { correct } \\
\text { erroné }\end{array}$ & $\begin{array}{c}10 \\
0\end{array}$ & $\begin{array}{c}14 \\
0\end{array}$ & $\begin{array}{l}0 \\
0\end{array}$ & $\begin{array}{c}24 \\
0\end{array}$ \\
\hline $\begin{array}{l}\text { Doc. } 7 \text { Code pénal } \\
\text { correct } \\
\text { erroné }\end{array}$ & $\begin{array}{c}20 \\
1\end{array}$ & $\begin{array}{c}31 \\
0\end{array}$ & $\begin{array}{l}7 \\
1\end{array}$ & $\begin{array}{c}58 \\
2\end{array}$ \\
\hline $\begin{array}{l}\text { Total général } \\
\text { correct } \\
\text { erroné }\end{array}$ & $\begin{array}{c}102 \\
9\end{array}$ & $\begin{array}{c}145 \\
4\end{array}$ & $\begin{array}{c}32 \\
2\end{array}$ & $\begin{array}{c}279 \\
15\end{array}$ \\
\hline
\end{tabular}

Tableau 5 : Nombre d'occurrences des mots (occurrences globales)

On constate une forte réutilisation de mots sur lesquels l'attention a été focalisée, et cela dans une proportion remarquable d'environ $\mathbf{9 5 \%}$ d'emplois corrects.

\section{Modes d'intégration des documents ressources}

Dans cette partie, nous examinerons en détail les configurations textuelles de l'intertextualité. Nous nous pencherons ainsi sur les différentes matérialisations de la présence, dans les textes des étudiants, des documents ressources. Cette présence laisse des traces que nous catégorisons de la manière suivante :

D’abord il y a les cas de réutilisation de lexèmes (telle qu'elle a été examinée quantitativement ci-dessus).

Il peut arriver aussi que les scripteurs «dépassent» le niveau lexical et intègrent en une configuration syntaxique originale des éléments prélevés dans différents syntagmes: dans ce cas nous parlerons de reconfiguration syntaxique d'éléments lexicaux. 
Thérèse Jeanneret, Raymond Capré et Dominique Vernaud

Troisièmement, nous nous attacherons aux cas où les scripteurs recourent à la citation : nous considérerons comme citation uniquement les cas où le segment est marqué par des guillemets et repérables par nous dans un texte ressource ${ }^{7}$.

Enfin nous étudierons les cas où il n'y a pas de reprise formelle d'un élément mais résumé, condensation en une formulation d'un argument, d'un exemple, etc. d'un des textes ressources (ce cas a été examiné quantitativement ci-dessus sous la rubrique allusion).

Ces quatre types de traces d'intertextualité sous-tendent, à notre avis, trois opérations. D’abord une opération de repérage : les étudiants sont amenés à identifier, dans les textes ressources, des lexèmes, des segments textuels, des configurations syntaxiques ou des idées, arguments. Dans un second temps, ils vont les prélever, c'est-à-dire segmenter dans le texte ressource l'élément pertinent. Cette opération de prélèvement doit s'accompagner d'une analyse du contexte pour extraire l'ensemble des informations nécessaires à la recontextualisation : genre (s'il s'agit d'un lexème), dépendance rectionnelle au sein du segment textuel (complétude de la séquence) et entre ce segment et son environnement, fonctionnement de la configuration syntaxique. Enfin, dans un troisième temps et en fonction des informations recueillies précédemment, il leur faudra procéder à l'opération d'insertion et d'intégration dans leur propre texte.

Nous allons maintenant présenter quelques exemples permettant d'observer comment se réalisent ces différentes opérations et les difficultés qu'elles soulèvent.

\section{Les prélèvements formels}

Une première série d'exemples (de (1) à (8)) mettra en évidence le travail de textualisation auquel doit se livrer le scripteur, même dans les cas où il ne semble prélever qu'un lexème.

L'exemple (1) montre ainsi la reprise d'une expression liée au thème de l'autodéfense: pléthore d'armes à feu. Le contexte d'occurrence de l'expression est modifié par la reformulation dans notre pays/dans un pays comme la Suisse. Il y a là une recontextualisation qui fait intervenir des critères plutôt énonciatifs :

\footnotetext{
${ }^{7}$ Nous laissons de côté les cas où les textes des étudiants présentent des segments textuels provenant littéralement d'un document ressource sans marque de citation. L'occurrence de ces citations masquées est, à notre sens, à mettre en rapport avec les «insuffisances» de nos consignes et notamment au flou concernant l'identité de l'auditoire.
} 
$(1)^{8}$

\begin{tabular}{|l|l|}
\hline Doc6 & Lb2 \\
\hline $\begin{array}{l}\text { Le constat qu'il dresse est sévère : il y a a } \\
\text { dans notre pays pléthore d'armes à feu. }\end{array}$ & $\begin{array}{l}\text { De surcroît, dans un pays comme la } \\
\text { Suisse, il y a pléthore d'armes à feu[...]. }\end{array}$ \\
\hline
\end{tabular}

L'exemple (2) met en évidence un cas il n'y a pas de recontextualisation énonciative. Cela pose au lecteur un problème pour interpréter notre pays. En effet, la scriptrice est slovaque !

(2)

\begin{tabular}{|l|l|}
\hline Doc6 & Lb9 \\
\hline «Notre pays est surarmé », affirme le & Même d'après le criminologue lausannois \\
criminologue lausannois Martin Killias. & Martin Killias, « notre pays est surarmé », \\
& ce n'est pas une bonne idée de laisser les \\
& habitants s'armer comme par exemple aux \\
& Etats-Unis. \\
\hline
\end{tabular}

On se trouve devant un cas envisagé par Moirand (1979) où un texte, produit dans une certaine situation d'écrit doit être modifié pour s'adapter à une autre situation d'écrit. En effet, l'occurrence du déterminant possessif notre est lié à la situation d'énonciation qui préside à l'interview dont est extrait le segment. La difficulté rencontrée par la scriptrice tient à son choix de segmenter la proposition complète et de la recontextualiser en citation. Il aurait été plus facile pour elle de se limiter à prélever le lexème surarmé.

Ainsi son choix de l'un ou l'autre type de prélèvement peut mettre le scripteur devant des difficultés qu'il n'est pas à même de résoudre.

L'exemple (3) montre la reprise dans une seule construction de plusieurs lexèmes disséminés dans un même article qui sont agencés en une configuration syntaxique originale :

(3)

\begin{tabular}{|l|l|}
\hline Doc 1 & Lb1 \\
\hline $\begin{array}{l}\text { Un chauffeur de taxi est assailli par des } \\
\text { jeunes }[\ldots] . \\
{[\ldots] \text { un ouvrier était tabassé }[\ldots]}\end{array}$ & $\begin{array}{l}\text { On oublie des règles quand on est agressé } \\
\text { ou tabassé ou assailli ou violenté. }\end{array}$ \\
\hline
\end{tabular}

\footnotetext{
${ }^{8}$ Les exemples sont présentés de la manière suivante: la colonne de gauche reproduit l'extrait pertinent du texte ressource (les documents ressources sont repérés par le numéro qu'ils portent en annexe 1), la colonne de droite, le texte de l'étudiant ( $\mathrm{La}, \mathrm{Lb}$ ou $\mathrm{N}$ indiquant le cours fréquenté, le chiffre symbolisant l'identité du scripteur). Par ailleurs, les fautes d'orthographe et les erreurs vénielles et non pertinentes pour le propos sont corrigées.
} 
Du point de vue des opérations de saisie auxquelles se livrent les scripteurs pour reprendre dans leur texte un segment - et notamment un lexème, il faut relever que l'on ne peut simplement les ramener à une opération de copie. En effet, il est vraisemblable que les scripteurs dissocient, en tout cas dans certains cas, lecture des documents et rédaction. Des exemples de confusions paronymiques comme (4) et (5) plaident pour cette idée. Comme le fait remarquer Nyckees (1998) quand il se penche sur les causes internes du changement de sens, les attractions paronymiques ne se réalisent qu'en contexte: pour (4), l'adjectif corporel favorise probablement la confusion 9 !

(4)

\begin{tabular}{|l|l|}
\hline Doc1 & Lb15 \\
\hline $\begin{array}{l}\text { Il se trouve aujourd'hui prévenu de } \\
\text { lésions corporelles graves. }\end{array}$ & $\begin{array}{l}\text { Le chauffeur de taxi qui s'est défendu avec } \\
\text { une baïonnette s'est retrouvé « prévenu de } \\
\text { liaisons corporelles graves ». }\end{array}$ \\
\hline
\end{tabular}

Le même commentaire peut être fait à propos de (5): le péril doit également être éminent pour que l'autodéfense soit légitime!

(5)

\begin{tabular}{|l|l|}
\hline Doc7 & Lb4 \\
\hline $\begin{array}{l}\text { Celui qui est attaqué sans droit ou menacé } \\
\text { sans droit d'une attaque imminente a le } \\
\text { droit de repousser l'attaque ... }\end{array}$ & $\begin{array}{l}\text { Dans une situation de péril éminent, } \\
\text { l'homme réagit comme un animal. }\end{array}$ \\
\hline
\end{tabular}

L'exemple (6) présente, outre des réutilisations de mots contenus dans le document 1 et dans la fiche de préparation qui s'y rapporte, le mot assaillant qui ne se trouve ni dans le texte ni dans la fiche de préparation.

(6)

\begin{tabular}{|c|c|}
\hline Doc 1 & La3 \\
\hline $\begin{array}{l}\text {...un jeune chauffeur d'Aubonne se faisait } \\
\text { assaillir devant un café-restaurant... } \\
\text { Le jeune Aubonnois se défendait au moyen } \\
\text { d'une baionnette }\end{array}$ & $\begin{array}{l}\text { Rappelons-nous le cas du chauffeur de taxi } \\
\text { d'Aubonne qui s'est fait assaillir par des } \\
\text { jeunes et qui s'est défendu avec une } \\
\text { baïonnette. Aujourd'hui c'est lui qui est } \\
\text { accusé parce qu'il a blessé un de ses } \\
\text { assaillants. }\end{array}$ \\
\hline
\end{tabular}
d'assaillir.

Il pourrait donc s'agir d'une bonne réussite de dérivation lexicale faite à partir

\footnotetext{
${ }^{9}$ Interrogée sur les raisons possibles de la confusion entre lésion et liaison, l'étudiante n'a tout d'abord pas remarqué l'erreur! Puis elle a reconnu n'avoir tout simplement pas compris le mot lésion...
} 
La série de l'exemple (7) montre différentes textualisations de l'expression (elle aussi tirée du document 1) : de victime, le chauffeur de taxi s'est retrouvé agresseur :

\begin{tabular}{|c|c|c|c|c|}
\hline N6 & N9 & N3 & N7 & $\mathrm{La} 7$ \\
\hline $\begin{array}{l}\text { C'est surtout le } \\
\text { fait d'utiliser } \\
\text { une arme qui a } \\
\text { fait de lui non } \\
\text { pas une } \\
\text { victime mais } \\
\text { un agresseur }\end{array}$ & $\begin{array}{l}\text { Pour ne pas } \\
\text { arriver à passer } \\
\text { d'un état de } \\
\text { victime à celui } \\
\text { d'agresseur. } \\
\text { [plus loin] } \\
\text { passer d'une } \\
\text { situation de } \\
\text { victime à une } \\
\text { autre } \\
\text { d'agresseur. }\end{array}$ & $\begin{array}{l}\text { Au lieu d'être } \\
\text { l'agressé on } \\
\text { devient } \\
\text { l'agresseur }\end{array}$ & $\begin{array}{l}\text { Il a été } \\
\text { d'abord } \\
\text { agressé mais } \\
\text { enfin, il s'est } \\
\text { retrouvé } \\
\text { agresseur. }\end{array}$ & $\begin{array}{l}\text { Une victime, } \\
\text { aujourd'hui peut } \\
\text { facilement se } \\
\text { trouver dans la } \\
\text { position } \\
\text { d'agresseur, } \\
\text { même si cette } \\
\text { personne a été, } \\
\text { elle, agressée. }\end{array}$ \\
\hline
\end{tabular}

Dans le document ressource, la paire de noms réciproques victime-agresseur est contextualisée dans un processus dynamique : le passage de victime à agresseur. On remarquera donc que certains scripteurs se limitent à la réutilisation des deux lexèmes (c'est le cas de N6), tandis que d'autres tentent également d'exprimer la dynamique du processus (c'est le cas de N9, N3 et N7). Par ailleurs, on trouve, dans différents travaux, le lexème agressé qui vient remplacer le terme de victime. Cette manière d'exploiter un des termes pour en produire un second est comparable à ce qui a été vu dans l'exemple (6). Beaucoup de scripteurs (l'exemple (7) ne reproduit pas exhaustivement l'ensemble des reprises de ce couple de termes) lient ainsi morphologiquement agent et patient et manifestent par là leur maîtrise d'une opposition morphologico-sémantique du français. Le scripteur La7 emploie de son côté le verbe agresser et il reprend le couple victime-agresseur. Cet exemple (7) met en évidence l'intrication de différents niveaux de construction du texte : on peut y voir se résoudre des problèmes de linéarisation du processus de victime à agresseur (passer, devenir, etc. avec la maitrise des rections afférentes) et des effets argumentatifs. En effet, ce segment de texte a un intérêt argumentatif évident en ce qu'il permet de discuter un aspect des problèmes posés par la notion d'autodéfense, susceptible, justement, d'amener une victime à devenir agresseur.

L'exemple (8) - outre la réutilisation du lexème septuagénaire - permet d'observer d'une part une reformulation de l'expression un peu après minuit en vers minuit ce qui dénote la maitrise des deux expressions et de leur correspondance et d'autre part celle de la diathèse du verbe importuner. 
(8)

\begin{tabular}{|l|l|}
\hline Doc3 & N7 \\
\hline $\begin{array}{l}\text { un septuagénaire... } \\
\text { un peu après minuit... } \\
\text { il a importuné son voisin de palier }\end{array}$ & $\begin{array}{l}\text { Un septuagénaire a tiré sur son voisin et } \\
\text { l'a tué juste parce qu'il a été importuné } \\
\text { par l'autre vers minuit }\end{array}$ \\
\hline
\end{tabular}

On notera que cette recontextualisation permet au scripteur de réussir la linéarisation de son paragraphe en assurant la progression de l'information par thème constant : le septuagénaire est le thème des différents prédicats. Cette construction à thème constant et le passif qui la sous-tend impose l'expression de l'agent: par ce dernier aurait été plus élégant que par l'autre qui parait un peu maladroit ici.

Cette réussite dans l'identification de l'agresseur et de l'agressé nous permet de relever une difficulté réelle : pour se référer aux quatre faits divers proposés, il fallait être capable de présenter clairement les protagonistes du fait divers et pour ce faire maitriser les différents procédés textuels de référence et d'anaphore nécessaires. L'exemple (9) met en évidence les difficultés rencontrées quand cette maittrise n'est pas sans faille :

(9)

\begin{tabular}{|c|c|}
\hline Doc 3 & La 9 \\
\hline $\begin{array}{l}\text { La réaction du voisin, un septuagénaire, } \\
\text { fut de sortir armé d'une carabine. Selon ses } \\
\text { déclarations, le coup de feu serait parti lors } \\
\text { de l'empoignade avec son voisin. }\end{array}$ & $\begin{array}{l}\text { Il y a deux voisins. Une nuit, l'un des } \\
\text { deux entend un bruit de la manière } \\
\text { quelqu'un frappant contre sa porte. Il, un } \\
\text { septuagénaire, sort sa carabine et tire à } \\
\text { l'homme... }\end{array}$ \\
\hline
\end{tabular}

Les exemples suivants (de (10) à (13)) permettront de voir que la textualisation peut se déployer sur des fragments de textes plus longs que ce que nous avons vu jusqu'ici.

Dans l'exemple (10), le scripteur réutilise plusieurs éléments contenus dans différents documents (les documents 7 (extrait de la loi) et 1 (Se défendre? Oui mais sans arme)). Tout d'abord, il organise une opposition, au moyen de bien que, de ce que la loi autorise - le port d'arme - et des recommandations de la police. Ces recommandations sont introduites par les verbes conseiller/déconseiller correctement utilisés sur le plan rectionnel: une fois en utilisant la structure $N V a ̀$ NdeV, une deuxième fois au moyen de $N V$ deV et deV, et une troisième fois au moyen de $N V N$. Ainsi, les notions contenues dans les documents - ne pas utiliser d'armes, fuir, avertir la police, éviter les armes factices - sont-elles reprises par le scripteur qui les a réutilisées dans un environnement syntaxique modifié et parfaitement correct. 
(10)

\begin{tabular}{|c|c|}
\hline Doc $7+\operatorname{Doc} 1$ & $\mathrm{La} 4$ \\
\hline $\begin{array}{l}\text { Celui qui est attaqué sans droit ou menacé } \\
\text { sans droit d'une attaque imminente a le } \\
\text { droit de repousser l'attaque par des moyens } \\
\text { proportionnés aux circonstances...(doc } 7) \\
\text { en cas de danger, ne pas chercher le conflit, } \\
\text { mais fuir si possible et avertir } \\
\text { rapidement la police. (doc 1) } \\
\text { Même les armes à feu factices sont à éviter, } \\
\text { car si l'agresseur brandit une arme réelle, la } \\
\text { vue d'un simple pistolet d'alarme (ou en } \\
\text { plastique) peut l'inciter à tirer. }\end{array}$ & $\begin{array}{l}\text { Bien que la loi n'interdise pas aux civils } \\
\text { de posséder une arme, la police } \\
\text { déconseille aux citoyens de se } \\
\text { défendre de telle manière et de choisir } \\
\text { une forme agressive pour résoudre des } \\
\text { problèmes. L'autorité conseille plutôt } \\
\text { de s'échapper (si on peut) et d'appeler } \\
\text { la police le plus tôt possible. [...] Et elle } \\
\text { déconseille surtout l'utilisation des } \\
\text { armes à feu factices parce qu'elles } \\
\text { peuvent inciter l'agresseur à tirer en } \\
\text { créant une situation encore plus sérieuse. }\end{array}$ \\
\hline
\end{tabular}

Remarquons aussi la réutilisation de l'expression inciter à tirer (document 1) devenue dans la recontextualisation elles (= les armes factices) peuvent inciter l'agresseur à tirer.

L'exemple (11) permet de souligner la dispersion des emprunts textuels et des modes de prélèvement auxquels recourent les scripteurs :

(11)

\begin{tabular}{|l|l|}
\hline Doc1 + doc5 & La1 \\
\hline De plus en cas de danger, ne pas chercher & Si j'étais personnellement confronté avec \\
le conflit, mais fuir si possible et avertir & un malfaiteur potentiel, je voudrais aussi \\
rapidement la police. & préférer de fuir et avertir la police. \\
Mais les appels à la police ne débouchent & Malheureusement on n'a pas toujours la \\
que sur une réponse : «Débrouillez- & $\begin{array}{l}\text { possibilité d'échapper du danger. A travers } \\
\text { vous». }\end{array}$ \\
le monde il y a beaucoup trop d'endroits \\
où la police n'arrive pas quand on les \\
appelle, comme c'est le cas pour Les gens \\
d'en dehors en Haïti.
\end{tabular}

Ici il y a emprunt à deux documents. Si l'on peut considérer que la première phrase reconfigure le segment textuel du document 1 - avec un problème de maîtrise de la rection du verbe préférer, la troisième renvoie au document 5 sur le mode du résumé. En suivant Vigner (1991), nous admettrons qu'il s'agit là d'une généralisation, permettant de retenir un aspect que les deux articles (par ailleurs fort différents) ont en commun, l'appel à la police.

Dans l'exemple (12), la scriptrice utilise la citation de la loi suisse pour construire son argumentation : 
(12)

\begin{tabular}{|c|c|}
\hline Doc7 + doc1 & $\mathrm{N} 4$ \\
\hline $\begin{array}{l}\text { «celui qui est attaqué sans droit ou menacé } \\
\text { sans droit d'une attaque imminente a le } \\
\text { droit de repousser l'attaque par des moyens } \\
\text { proportionnés aux circonstances. Le même } \\
\text { droit appartient à des tiers.» } \\
\text { [fuir si possible et avertir rapidement la } \\
\text { police] }\end{array}$ & $\begin{array}{l}\text { Selon la loi «celui qui est attaqué sans } \\
\text { droit ou menacé sans droit d'une attaque } \\
\text { imminente a le droit de repousser l'attaque } \\
\text { par des moyens proportionnés aux } \\
\text { circonstances. Le même droit appartient à } \\
\text { des tiers», donc si on se trouve dans une } \\
\text { situation de danger grave on peut utiliser } \\
\text { les moyens disponibles mais la police } \\
\text { conseille de prendre des précautions, fuir } \\
\text { si possible et avertir rapidement les } \\
\text { autorités. }\end{array}$ \\
\hline
\end{tabular}

Elle tire de la citation le droit de se défendre dans une situation grave. Mais elle nuance sa position en intégrant, cette fois sans citation mais en les reformulant, les conseils prodigués par la police lausannoise reproduits dans le document 1 . L'intégration syntaxique n'est pas parfaitement réussie parce que la scriptrice ne réitère pas la marque de complémentation de devant fuir puis devant avertir et restreint ainsi le marquage de la dépendance rectionnelle au premier terme ${ }^{10}$.

Les deux exemples suivants permettent d'insister sur le travail de fond - et sa difficulté parfois - que nécessite la recontextualisation:

(13)

\begin{tabular}{|l|l|}
\hline Doc5 & N1 \\
\hline $\begin{array}{l}\text { Les habitants du bidonville de Martissant, } \\
\text { à Port-au-Prince, ont mis sur pied une } \\
\text { myriade d'associations pour pallier autant } \\
\text { que faire se peut la carence totale des } \\
\text { services publics. }\end{array}$ & $\begin{array}{l}\text { contre la carti, les habitants se battent } \\
\text { publics. Leur «arme » }: \text { la coopération et la } \\
\text { mobilisation collective. }\end{array}$ \\
\hline
\end{tabular}

Dans cet exemple, la scriptrice intègre la carence totale des services publics à une construction originale qui reformule le segment textuel du document 5 . Elle choisit la métaphore de la bataille et est capable de la filer dans la seconde phrase de l'exemple par l'utilisation du lexème arme.

Dans l'exemple (14) en revanche, la scriptrice construit le prédicat être dans la carence totale des services publics. Son texte permet de faire l'hypothèse qu'elle a compris le sens global de l'expression mais qu'elle ne connait pas l'expression idiomatique antonyme de pallier la carence/souffrir de la carence :

\footnotetext{
${ }^{10}$ Dans Jeanneret (2002), plusieurs problèmes d'intégration en citation de ce même segment régi par le verbe conseiller sont présentés.
} 


\begin{tabular}{|l|l|}
\hline Doc5 & N4 \\
\hline Les habitants du bidonville de Martissant, & On peut discuter aussi la notion de \\
à Port-au-Prince, ont mis sur pied une & l'autodéfense dans un groupe comme le \\
myriade d'associations pour pallier autant & cas des habitants de bidonville de \\
que faire se peut la carence totale des & Martissant à Port-au-Prince; ces gens qui \\
services publics. & $\begin{array}{l}\text { habitent dans des conditions très } \\
\text { mauvaises car ils sont dans la carence } \\
\text { totale des services publics. }\end{array}$ \\
\hline
\end{tabular}

\section{Les prélèvements conceptuels}

A côté de tous ces prélèvements de segments textuels de quelque longueur qu'ils soient et quels que soient les modes précis de leur recontextualisation, nous avons répertorié beaucoup de cas, semblables aux exemples (10) et (11), où les scripteurs se sont référés aux documents ressources plutôt pour en extraire certaines informations représentées afin de les utiliser dans leurs argumentations. Nous ne nous intéresserons dans cette partie qu'aux cas où les scripteurs ont généralisé, globalisé certaines informations figurant dans les documents ressources : nous admettrons que les scripteurs opèrent là un résumé du document (ou d'un aspect du document) et nous considérerons avec Vigner (1991:35), que le résumé «peut être assimilé à une opération de généralisation, opération par laquelle on approche un objet singulier de l'objet générique de référence par perte au moins partielle, de certains éléments de spécification et de particularisation.»

Une des opérations constitutives, d'après Vigner, du résumé est ce qu'il appelle la globalisation, qui permet de condenser en une formulation à la fois plus générale et plus abstraite une suite d'informations de moindre importance. Ainsi, dans l'exemple (15), la scriptrice intègre par l'expression les banales disputes entre voisins causées par le bruit ou la fumée d'un gril les circonstances des faits divers rapportés dans les documents 3 et $4^{11}$.

(15)

\begin{tabular}{|l|l|}
\hline Doc3 et Doc4 & Lb5 \\
\hline Voir en annexe & $\begin{array}{l}\text { Donc, les banales disputes entre voisins, } \\
\text { causées par le bruit ou la fumée d'un gril, } \\
\text { ne méritent pas d'être prises en } \\
\text { considération. }\end{array}$ \\
\hline
\end{tabular}

Dans l'exemple (16), ce sont les opérations d'élimination des informations jugées peu importantes et d'intégration - ici dans une relative - des circonstances factuelles nécessaires à la compréhension du cas qui sont remarquables.

\footnotetext{
${ }^{11}$ Notons une globalisation très proche dans un autre texte d'étudiant : les disputes de voisinage.
} 
Thérèse Jeanneret, Raymond Capré et Dominique Vernaud

(16)

\begin{tabular}{|c|c|}
\hline Doc 2 & La 1 \\
\hline $\begin{array}{l}\text { Son geste héroïque remonte à un vendredi } \\
\text { soir du mois de novembre } 2000 \text {, lorsque } \\
\text { cette apprentie est sortie boire un verre } \\
\text { avec deux collègues dans un pub de son } \\
\text { village. } \\
\text { L'ambiance a tourné au vinaigre avec } \\
\text { l'intrusion d'une équipe de crânes rasés } \\
\text { inconnus au village. } \\
{[+6 \text { lignes }]} \\
\text { Hors d'elle, entre cris et pleurs, la } \\
\text { vendeuse de } 20 \text { ans a éloigné un agresseur } \\
\text { à coups de poing. }\end{array}$ & $\begin{array}{l}\text { Le cas de Sophie qui éloigne un crâne rasé } \\
\text { à coups de poing, après qu'il a frappé son } \\
\text { ami vietnamien jusqu'à ce qu'il perde } \\
\text { connaissance }[\ldots . . \text { est bien évidemment un } \\
\text { exemple où l'autodéfense se justifie. }\end{array}$ \\
\hline
\end{tabular}

Tout en réutilisant une partie du lexique: crâne rasé, à coups de poing, perdre connaissance, la scriptrice utilise ce résumé dans son argumentation en faveur de l'autodéfense. A la fin du passage, nous trouvons en effet: est un exemple où l'autodéfense se justifie. Le document fourni a donc permis à l'apprenante de faire un travail de résumé de texte et en même temps d'enrichir son texte d'un exemple concret.

Dans l'exemple (17), à propos du même document 2, la scriptrice nous fournit également un résumé pour appuyer sa critique envers la justice.

(17)

\begin{tabular}{|l|l|}
\hline Doc 2 & La 4 \\
\hline voir en annexe Doc2 & $\begin{array}{l}\text { La jeune vendeuse est obligée de payer une } \\
\text { amende parce qu'elle a frappé le skinhead } \\
\text { qui a attaqué son collegue vietnamien. }\end{array}$ \\
\hline
\end{tabular}

Il est vrai qu'elle s'appuie également sur la formulation du titre du document: Sophie amendée pour avoir frappé un skinhead. Mais elle y intègre une relative qui-comme dans l'exemple (15) - permet de saisir les faits pertinents.

Dans l'exemple (18), le résumé permet à la scriptrice d'expliciter le double lien qui lie le document 5 à la problématique de l'autodéfense : en tant qu'il décrit un mode d'autodéfense que l'on pourrait appeler social, matérialisant des aspects de solidarité des habitants les uns vis-à-vis des autres et en tant qu'il présente un cas de dérapage de l'autodéfense, le lynchage de malfaiteurs relâchés par la police. 
$(18)$

\begin{tabular}{|l|l|}
\hline Doc5 & Lb7 \\
\hline Voir en annexe Doc5 & L'exemple de Martissant, en Haïti, est très \\
parlant. Là-bas, les gens oubliés et \\
marginalisés par l'Etat, «se définissent eux- \\
mêmes comme les gens du dehors ». De ce \\
fait, ils ont créé plusieurs associations pour \\
se défendre contre la pauvreté, l'inefficacité \\
de la police et le manque de services \\
publics. Ils ont mis sur pied un centre de \\
santé, le ramassage d'ordures et une \\
mutuelle de solidarité. [...] On comprend \\
alors mieux pourquoi ils ont leur propre \\
brigade de vigilance, afin d'éviter le \\
banditisme. Il arrive pourtant parfois que la \\
violence ambiante les oblige à recourir à \\
des actes extrêmes, comme le lynchage de \\
personnes relâchées par la police.
\end{tabular}

En conclusion de cette partie, on constate donc que les données prélevées par les scripteurs leur offrent deux types de ressources : en matériel linguistique, d'une part et en contenu, d'autre part, même s'il va de soi que cette distinction est en soi impossible et que les prélèvements formels peuvent se révéler porteurs d'idées exploitables, tandis que les prélèvements conceptuels offrent également et en même temps des ressources linguistiques. Il n'en reste pas moins que ces deux types de ressource sont centraux pour la rédaction d'un texte aussi bien théorique que délibératif : les prélèvements conceptuels imposent au scripteur de prendre distance par rapport aux documents ressources, de se les approprier un peu plus, les prélèvements formels lui imposent une gestion locale de l'intertextualité dont on a vu qu'elle n'était pas toujours évidente.

\section{Pistes pour une didactique du texte délibératif}

De toute évidence, l'expérience décrite dans les pages qui précèdent est réussie. Les exemples d'utilisation adéquate des documents sont largement plus nombreux que ceux des échecs. Le vocabulaire fourni par les documents et par les fiches de préparation est remarquablement bien utilisé. Les textes des étudiants, par la richesse de leur argumentation et l'abondance des exemples puisés dans les documents, présentent une qualité qui nous encourage à poursuivre dans cette voie. Resterait à les comparer à des textes produits sur le même sujet par des étudiants de mêmes niveaux qui n'auraient pas reçu les documents, pour pouvoir établir avec certitude l'efficacité de cette façon de travailler le texte délibératif. L'absence d'une telle comparaison ne nous empêche pas de recommander l'approche décrite dans ces pages. Les pistes 
didactiques qui s'ouvrent devant nous pour continuer à enseigner la rédaction du texte délibératif sont riches.

Du point de vue acquisitionnel, on peut espérer, nous semble-t-il, qu'un certain nombre de lexèmes, de constructions, aient été acquis à l'issue de l'exercice. L'ensemble des opérations de repérage, prélèvement et insertion évoquées plus haut pourraient valoir comme une sorte de version écrite et monologale de la séquence potentiellement acquisitionnelle telle qu'elle a été développée à partir de l'article de Pietro, Matthey \& Py (1989).

Complétons ce constat en fournissant quelques suggestions qui devraient permettre de continuer de telles expériences tout en les améliorant.

\section{Quels thèmes, quels documents?}

Il va de soi que le thème doit correspondre aux intérêts des apprenants, particulièrement dans une didactique centrée sur l'apprenant. Pour cela plusieurs moyens sont possibles : le choix du thème en classe, par exemple, sur la base d'une discussion libre ou de listes élaborées par l'enseignant, ou d'une recherche parmi les titres de l'actualité de la semaine. Il serait particulièrement important de trouver un thème inattendu ou relativement restreint; les grands thèmes connus du genre «la peine de mort», «l'euthanasie», «l'avortement», ou encore "l'énergie nucléaire», trop vastes et trop souvent exploités ne provoquent souvent que des réactions de lassitude ou alors des stéréotypes.

Une fois le thème choisi, il convient de trouver une collection de documents adéquats. Il nous a paru intéressant - et productif - de pouvoir fournir des documents variés: témoignages divers, récits, réflexions, analyses, ou même des documents techniques, comme dans notre cas les articles de la loi. Cette recherche n'est pas toujours aisée. L'aide des étudiants pourrait s'avérer fructueuse, ou alors le travail d'équipe.

\section{Niveau des apprenants}

Dans le cas de notre expérience, le niveau des apprenants s'inscrivait dans une fourchette allant de A2 à C1, selon les échelles de compétence du Conseil de l'Europe. Compte tenu du thème choisi et des documents fournis, les niveaux B2 et $\mathrm{C} 1$ étaient privilégiés. Toutefois, la méthode de travail pourrait convenir à tous les niveaux à partir de A2. Dans ce cas, il conviendrait de choisir les documents avec soin. On peut imaginer des documents fabriqués pour le niveau A2. La méthode Tempo ${ }^{12}$, par exemple, nous fournit de bons modèles de documents fabriqués et/ou authentiques qui servent de point de départ à la production de textes écrits.

\footnotetext{
12 Bérard, E., Canier, Y., Lavenne, C., Tempo 2, Paris, Les Editions Didier, 1997. On consultera avec intérêt le chapitre 7, «Argumenten», ou le chapitre 5, «Rapporter les paroles de quelqu'un».
} 


\section{Destinataires des textes}

La question du destinataire du texte, ou des destinataires, nous parait cruciale. Certes, nous sommes en situation scolaire ou universitaire. Le but du travail est d'écrire un «bon» texte qui sera en fin de compte évalué par l'enseignant. Mais il faudrait éviter que, au premier degré, le destinataire ne soit que l'enseignant. Si l'on part du principe que l'apprentissage du texte délibératif sera utilisé pour défendre un point de vue ou présenter un problème à débattre, le scripteur pourra orienter son texte de façon beaucoup plus cohérente s'il a imaginé ses destinataires. Parmi de nombreuses possibilités, mentionnons, à titre d'exemples, quelques consignes qu'il serait possible de proposer :

- Texte à produire dans le cadre d'un groupe de travail, avec le présupposé que chaque participant a lu les documents fournis.

- Texte produit par un journaliste qui écrit à la suite de quelques cas connus de la majorité des lecteurs.

- Texte produit par un journaliste d'un magazine en présupposant que les cas ne sont guère connus des lecteurs.

- Réponse à un lecteur qui aurait pris clairement telle ou telle position.

Le fait d'insister sur ce point permettrait de lever des ambiguités. D'une part l'apprenant devrait prendre conscience que l'on écrit pour des destinataires particuliers, d'autre part il verrait plus clairement de quelle façon réutiliser les documents : simples rappels, ou résumés des situations, ou même développements des situations dans les cas où l'on s'adresserait à un public qui n'en a jamais entendu parler. Dans l'expérience que nous avons conduite, nous n'avons peut-être pas suffisamment insisté sur cet aspect, si bien que nous avons constaté un certain flottement, certains scripteurs ne sachant pas très bien s'il fallait raconter certains événements ou s'ils étaient connus du destinataire. L'exemple suivant montre combien cette imprécision nuit à l'autonomie du texte de l'étudiant. Dans l'exemple (19), l'étudiante fait directement référence à un parcours des différents textes ressources :

(19)

\section{$\mathrm{N} 8$}

«[...] l'autodéfense implique différents aspects : si jusqu'à maintenant on a eu des cas où la violence est résolue parfois par la violence et avec des armes - cas présenté dans les pays bien développés économiquement - on passe à un pays fondé par des esclaves, Haït terre accomplissant «les gens d'en dehors»

Elle sous-entend clairement ainsi que son auditoire a non seulement connaissance des textes mais dispose de surcroît du même ensemble de documents et peut s'y référer dans le même ordre qu'elle. On note la même stratégie reposant sur la même hypothèse d'un auditoire disposant du même recueil de textes ressources chez plusieurs étudiants quand ils cherchent à introduire un aspect plus solidaire de l'autodéfense en évoquant l'article concernant Haïti (document 5). 
Il faut donc insister sur l'importance des consignes dans ce type d'activités en soulignant l'aspect structurant et potentiellement facilitant des contraintes qu'elles imposent aux scripteurs.

\section{Planification des activités}

Dans la partie II, Déroulement de la recherche, nous avons déjà indiqué les différentes articulations des travaux préliminaires, avant de lancer nos étudiants dans la rédaction de leurs textes. Il nous parait important de bien planifier ces travaux préliminaires, afin que chaque document fourni ait une importance égale, que l'on maintienne un bon équilibre entre la découverte personnelle d'un document et son exploitation à l'aide du questionnaire et que l'on ait le temps de mettre en évidence les éléments lexicaux dont on espère l'acquisition. Relevons encore que la méthodologie adoptée demande aux étudiants un gros effort de lecture et de compréhension des textes.

Il conviendra aussi de fixer clairement les règles du jeu : les documents fournis devront-ils être obligatoirement utilisés, et si oui y aura-t-il un minimum et un maximum de réutilisations possibles ? Quelle sera la dimension du travail à produire? Règles du jeu qui s'ajouteront à celle mentionnée plus haut : le choix de destinataires.

\section{Moyens techniques à enseigner}

L'utilisation d'une telle méthode d'enseignement du texte délibératif devrait s'accompagner d'un enseignement spécifique de certains éléments du texte à produire. Il faudrait imaginer une série d'activités destinées à entraîner d'une part, le prélèvement (avec l'analyse de son contexte) et d'autre part, l'insertion. L'enseignement du résumé et de la citation seraient incontournables. A noter que l'ensemble de cette problématique du texte comme ressource pour un autre texte impose également de réfléchir avec les étudiants aux limites entre ce qui peut simplement être réutilisé et ce qui doit être honnêtement attribué à son auteur. Les modes de prélèvement doivent ainsi être contraints par les recontextualisations envisagées et par leur étendue et leur importance. Cette réflexion a une grande importance tant dans la didactique du texte délibératif que dans celle du texte théorique.

L'ensemble de ces activités devrait servir à tirer parti des documents proposés. Il s'agirait ainsi d'en profiter pour aborder les éléments constitutifs du texte et les différentes techniques qui en permettent une bonne utilisation.

Remarquons enfin que l'enseignement du texte délibératif ne concerne pas que les cours d'expression écrite. Cet enseignement sera avantageusement renforcé si on peut l'articuler à d'autres cours, comme ce fut le cas, lors de notre expérience, pour le cours consacré à la revue de presse hebdomadaire. 


\section{Conclusion}

Au terme de cette étude, il nous faut revenir sur l'importance du choix des documents ressources. Il nous parait nécessaire d'en diversifier au maximum tant les genres discursifs que les supports substantiels. Des documents sonores, tels qu'extraits d'émissions de radio, débats, etc. devraient ainsi également pouvoir être proposés. La classe de langue pourrait d'ailleurs être utilisée comme pourvoyeuse de documents ressources par l'organisation de débats autour du thème de délibération choisi. Nous rejoindrions là des propositions faites par Nonnon (1996) qui montre que l'activité d'énonciation et ce qu'elle appelle le cheminement des échanges sont un facteur de prise de conscience et de transformation des attitudes épistémiques des sujets. Cette auteure insiste également sur la diversité des sources discursives traitées pour produire une argumentation.

Un deuxième aspect de cette étude nous parait offrir des pistes de réflexion : il s'agit de la manière dont on pourrait permettre à des étudiants alloglottes, tout en leur offrant des données pour la construction d'un univers sémiodiscursif en français, d'utiliser également leurs connaissances du thème de délibération et leurs ressources en langue maternelle. Il va de soi qu'il ne s'agirait pas de leur demander de traduire leurs références discursives mais de les modifier, de manière à ce qu'elles puissent venir éclairer, nuancer, etc. les données en français et leur donner l'occasion de développer un savoir-faire interprétatif de leur environnement. Il y aurait là une piste à développer dans un enseignement plurilingue.

Enfin - et c'est le point central - cette étude nous amène à défendre l'importance pour l'élaboration d'une didactique de l'écriture du texte en langue étrangère et d'une didactique du texte théorique de ce qu'on pourrait appeler une didactique de la ressource. Il faut en effet sensibiliser les apprentis scripteurs à l'intérêt et à la richesse de leur environnement discursif. Il faut leur enseigner à repérer, prélever, intégrer des formulations et des idées, à identifier des indices énonciatifs, à traiter les événements langagiers auxquels ils sont confrontés comme des ressources discursives susceptibles de leur permettre de construire de nouvelles compétences. De ce point de vue, cette étude met en évidence un mode d'articulation des savoirs scolaires et extrascolaires, un point de contact entre apprentissage et acquisition. 
Thérèse Jeanneret, Raymond Capré et Dominique Vernaud

\section{BIBLIOGRAPHIE :}

[Reichler-]BÉguelin, Marie-José ; Monique Dénervaud \& Janine Jespersen (1988) Ecrire en français, Paris, Delachaux \& Niestlé.

BOCH, Françoise \& Francis Grossmann (eds) (2001) Apprendre à citer le discours d'autrui, LIDIL 24

BronCKART, Jean-Paul et al. (1985) Le fonctionnement des discours, Paris, Delachaux \& Niestlé.

De Pietro, Jean-François ; Marinette MAtThey \& Bernard Py (1989) « Acquisition et contrat didactique: les séquences potentiellement acquisitionnelles de la conversation exolingue », Actes des troisièmes rencontres régionales de linguistique, Université de Strasbourg.

GAUTHIER, Claude \& Thérèse JEANNERET (eds) (2000) Français langue étrangère en milieu homoglotte et alloglotte : quels enseignements pour quelles pratiques effectives, quelles pratiques effectives après quels enseignements?, Bulletin VLAS-ASLA 71.

JEANNERET, Thérèse (2002) Figures de dialogisme et complémentation verbale dans des productions textuelles en FLE, TRANEL 37, 145-162.

MOIRAND, Sophie (1979) Situations d'écrit, Paris, Hatier.

Montredon, Jacques (1995) «Pédagogie en contexte», Le français dans le monde. Recherches et applications, $94-99$.

NONNON, Elisabeth (1996) «Activités argumentatives et élaboration de connaissances nouvelles : le dialogue comme espace d'exploration », Langue française 112, 67-87.

NyCKEES, Vincent (1998) La sémantique, Paris, Belin.

SimONIN-GRumbaCH, Jenny (1975) «Pour une typologie des discours », in : J. Kristeva, J.-C. Milner et N. Ruwet, Langue, discours, société. Pour Emile Benveniste, Paris, Le Seuil, 85-121.

VIGNER, Gérard (1991) «Réduction de l'information et généralisation : aspects cognitifs et linguistiques de l'activité de résumé », Pratiques 72, 33-54. 